\title{
Fabrication of multi-analyte nanosensors for biological targets - Introduction to AquaNOSE project
}

\author{
Leo Schranzhofer ${ }^{1}$, Joni Leinvuo ${ }^{2}$, Peter Lieberzeit ${ }^{3}$ \\ 1 Profactor $\mathrm{GmbH}$, Im Stadtgut A2, 4400 Steyr, Austria, \\ 2 BioMensio Oy, Hermiankatu 6-8 H, Fl-33720 Tampere, Finland \\ ${ }^{3}$ University of Vienna, Währingerstraße 42, 1090 Vienna, Austria \\ Leo.schranzhofer@profactor.at, joni.leinvuo@biomensio.com, peter.lieberzeit@univie.ac.at
}

\begin{abstract}
Microfluidics is the multidisciplinary field dealing with the science and engineering of any system that manipulates fluids in microliter/micrometer scales. Methods in nanotechnology, prototyping and the integration of functional elements as well as macro- to nano features into one single device are very difficult, time consuming and expensive to realize with state-of-the-art fabrication techniques. The vision of AquaNOSE is to close the gap between conceptual ideas in nanotechnology and production for the commercial microfluidic microelectromechanical systems ( $\mu$-MEMS) market to foster novel products. AquaNOSE combines novel concepts in printed electronics, molecularly imprinted polymer fabrication and integrated circuitry fabrication with state of the art production schemes based on injection molding.
\end{abstract}

Key words: molecularly imprinted polymers, additive manufacturing, lab-on-a-chip, $\mu$ f-MEMS, mycotoxins.

\section{Introduction}

A basic microfluidic device comprises analysis chambers and reservoirs connected through micro-channels. A lab-on-a-chip (LOC) device, a subset of $\mu \mathrm{f}-\mathrm{MEMS}$, exhibits combinations of microfluidics, sensing and manipulating tools for controlling signal output.[1] The benefits of miniaturization are ranging from small volume analytics, small processing units, low energy consumption, parallelization of measurements and faster assays, besides others. However, there is a large gap between novel microfluidic concepts/ideas and their final translation to commercial markets and applications.

\section{Project Overview}

To increase the rate of translation of innovative uf-MEMS concepts into viable market products, AquaNOSE will tackle the following research topics: (1) Generating nanostructured receptor based on molecularly imprinted polymers (MIP); (2) customized sensor chip fabrication, based on bulk acoustic resonance, and bonding; (3) microfluidic device fabrication via combination of injection molding and inkjet printing and; (4) demonstration of usability of a final device.:

\begin{abstract}
Nanostructured receptor
In AquaNOSE, two MIP-based approaches will be developed and optimized for mycotoxin and antibiotic targets. The first strategy is based on "Proto-MIP", a novel route, developed by PROFACTOR GmbH and University of Vienna. It is based on a smart combination of conventional surface MIP and nano imprint lithography (NIL) processes. Here, a stamp is used for imprinting, containing the template. This stamp is coated with a "anchor group", a functional linking layer, capable of aligning to the template and binding to a polymeric resin. This presents the necessary self-assembly for MIP generation. This Pre-MIP layer is fused within a lithographic process with an already cross-linked polymer resin. This step combines the polymeric backbone layer with the linking group to yield MIP cavities. In a preliminary study by PROFACTOR and University of Vienna, e.coli bacteria were used to generate MIP layers in this way. An overview is presented in Fig. 1 that shows on the one hand the used stamp surface showing immobilized bacteria and on the other hand the imprinted surface with no attached bacteria (microscope image), but with successful generated MIP cavities (AFM image). The second MIP strategy is based on core-shell MIP particles.
\end{abstract}




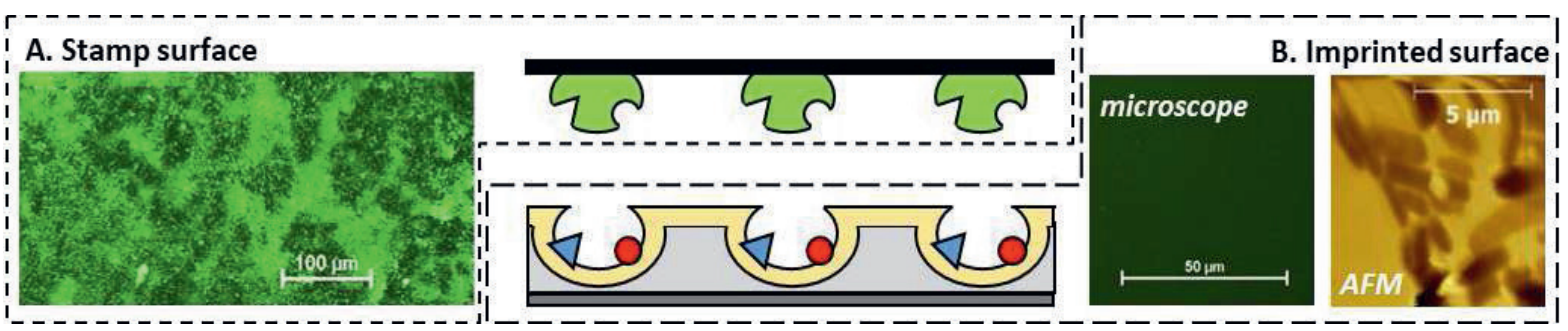

Fig.1. Proto-MIP example: A) Stamp surface with immobilized and marked bacteria (green), B) imprinted polymeric surface showing no bacteria transfer in microscope images but MIP cavities in AFM image

There, the particle shell represents the MIP layer, while the core is based on metal oxides. The idea behind the approach is to reverse the roles of analyte and MIPs, respectively, during signal generation. Target analyte is immobilized on the sensor surface and MIP particles are attached to them. When analyte concentration rises, core-shell MIP particles will gradually be released from the sensor surface causing a significant change in sensor signal. [2]

\section{Advanced microfluidic device}

Another main route within AquaNOSE is dealing with the fabrication of a microfluidic device by a clever combination of injection molding and additive manufacturing. An example is given schematically in Fig. 2. Electrically conductive ink is deposited via inkjet to fill cavities within the microfluidic device via capillary force.[3] Via this approach, feature sizes for electrically conductive parts below $20 \mu \mathrm{m}$ will be achieved by very cost-effective methods.

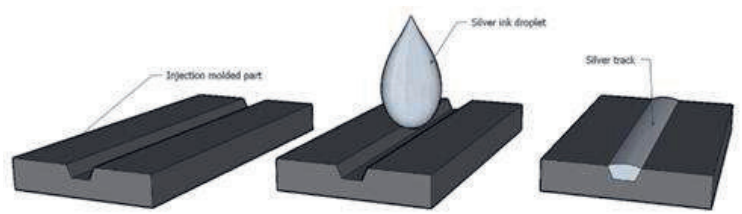

Fig. 2. Schematic representation of the steps to produce capillary filled electrically conductive tracks via injection molding and inkjet printing.

\section{Novel $\mu f-M E M S$ cartridge}

Finally, all individual parts will be assembled, including functionalized sensor chip as shown in Fig. 3, microfluidic cartridge and electrical paths to a fully functional sensing device. Designed by industrial partner BIOMENSIO [4] for their hand-held sensing tool, the device will be capable to detect mycotoxins in complex media, such as antibiotics in wastewater.

\section{Outlook}

Potential End Users are collaborating closely with the development within AquaNOSE. Exemplary, the resulting sensing system will be tested via a field study at a wastewater treatment plant at an antibiotic production facility.

\section{Acknowledgments}

This research was carried out within the "FTIProject DigiManu - Labor für die Digitalisierung der Produktion" project funded by the State of Upper Austria through the Strategic Economic and Research Program "Innovatives OÖ 2020" and the AquaNOSE project funded by the Austrian Research Promotion Agency (FFG) under Grand Agreement No. 864893. Furthermore, we would like to thank Annette Schnettelker and Vito Matteo Di Pietro for their effort in preliminary studies resulting in background knowledge for AquaNOSE.

\section{References}

[1] J. Berthier, P. Silberzan, Microfluidics for Biotechnology, Artech House, Boston, Mass., 2010. ISBN: 978-1-59693-443-6

[2] I. Vikholm-Lundin, S. Auer, A.-C. Hellgren, Sens. Actuators B Chem. 2011, 156, 28-34. DOI: 10.1016/j.snb.2011.03.069

[3] V. M. Di Pietro, M. Mühlberger, H. M. Außerhuber, SelfAligned, High Resolution Conductive Lines on Microstructured Imprinted Substrate (Poster), 43rd Micro- and Nano-Engineering conference, 2017, Braga, PT.

[4] http://www.biomensio.com/; accessed 01.02.2018

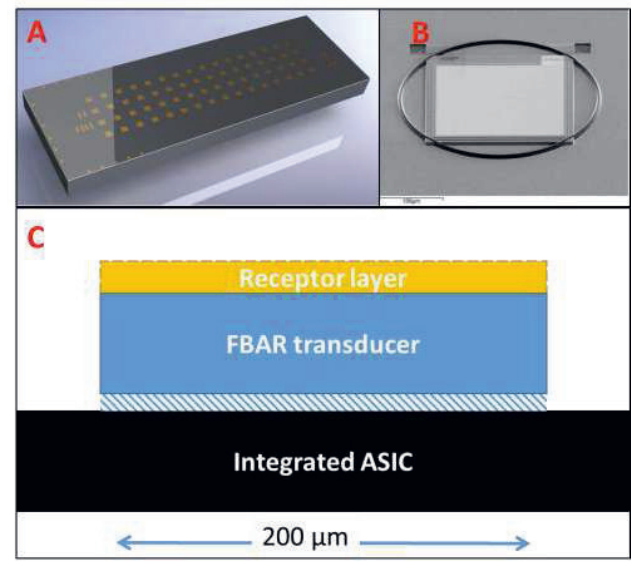

Fig. 3. Schematic overview of the sensor concept within AquaNOSE. A: sensor design by BIOMENSIO; $B$ : AFM image of a single sensor "pixel"; $C$ : schematic drawing of a single pixel containing a receptor layer, e.g. MIPs 